Boise State University

ScholarWorks

4-15-2019

\title{
A Time-Dependent Chemo-Mechanical Analysis of Alkali-Silica Reaction for the Disparate Geometry of Concrete Meso-Structure
}

Md. Asif Rahman

Boise State University

Yang Lu

Boise State University 


\section{A Time-Dependent Chemo-Mechanical Analysis of Alkali-Silica Reaction}

For The Disparate Geometry of Concrete Meso-Structure

RAHMAN, PROF. YANG LU*, Department of Civil Engineering

MicroMechanics \& Smart Infrastructure Group (MMIG)

MD. ASIF RAHMAN, PROF. YANG LU*, Depart
ITRODUCTION\& MOTIVATION ASR DAMAGE

Portland cement concrete is a common construction A threat to: material that is frequently used due to its durability, $>$ Concrete Pavement long service life and great economy. However, \& Bridges chemical reactions between reactive aggregate $>$ Nuclear Plant chemical reactions between reactive aggregate $>$ Nuclea
particles \& cement paste lead to concrete degradation over time. Alkali-silica reaction (ASR) is $>$ Airport Runway one of those undesirable chemical reactions. ASR $>$ Other Concrete damage is caused internally and becomes visible damage is caused internally and becomes visible when the structure is already damaged. It is a threat to socio-economic development of society. ASR is called as Concrete Cancer.

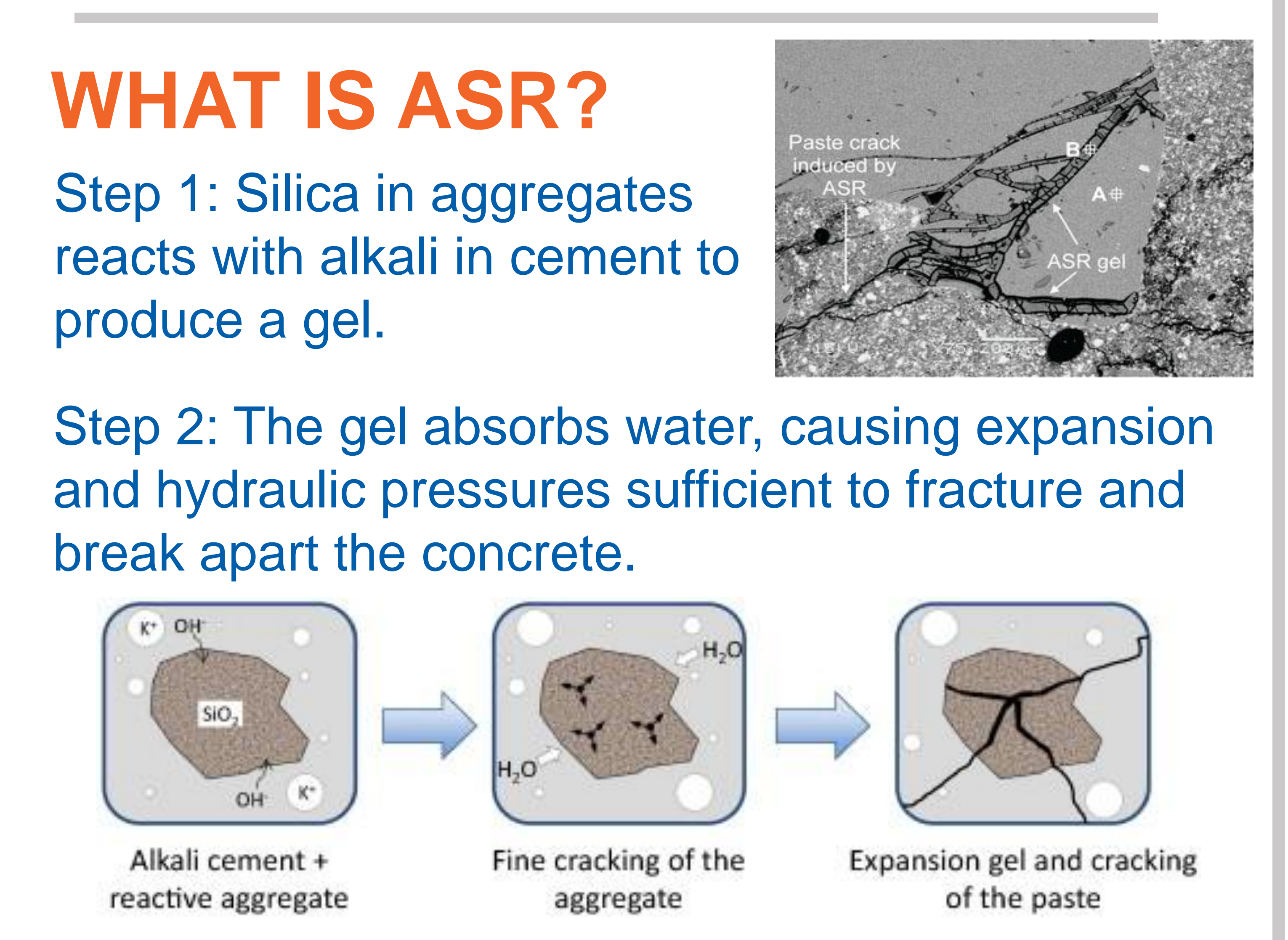

CNN Newsletter April 3, 2019

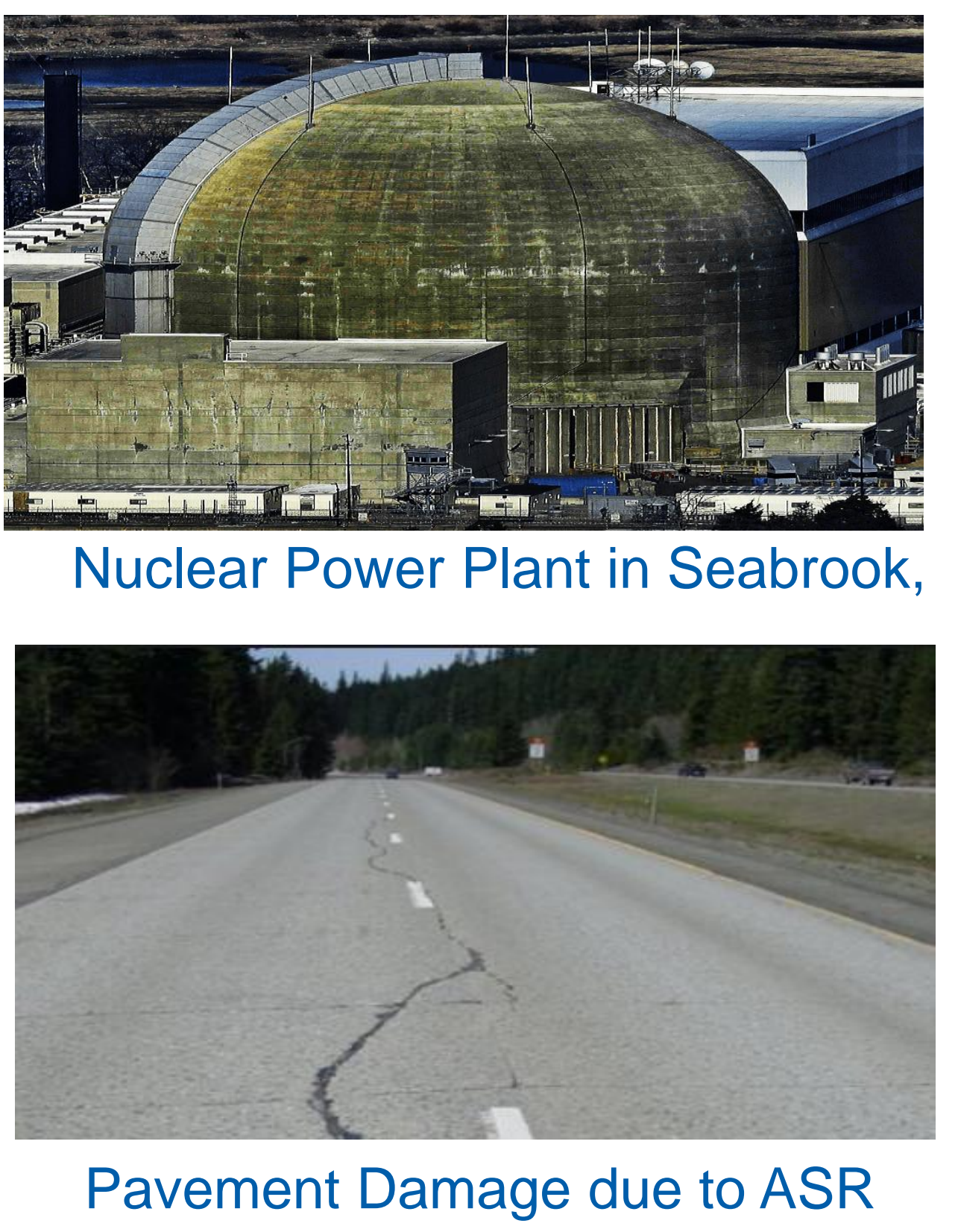

47,052 bridges need urgent repairs in USA.

- Rhode Island (23\%)

West Virginia (19.8\%)

lowa (19.3\%)

South Dakota (16.7\%)

Pennsylvania (16.5\%)

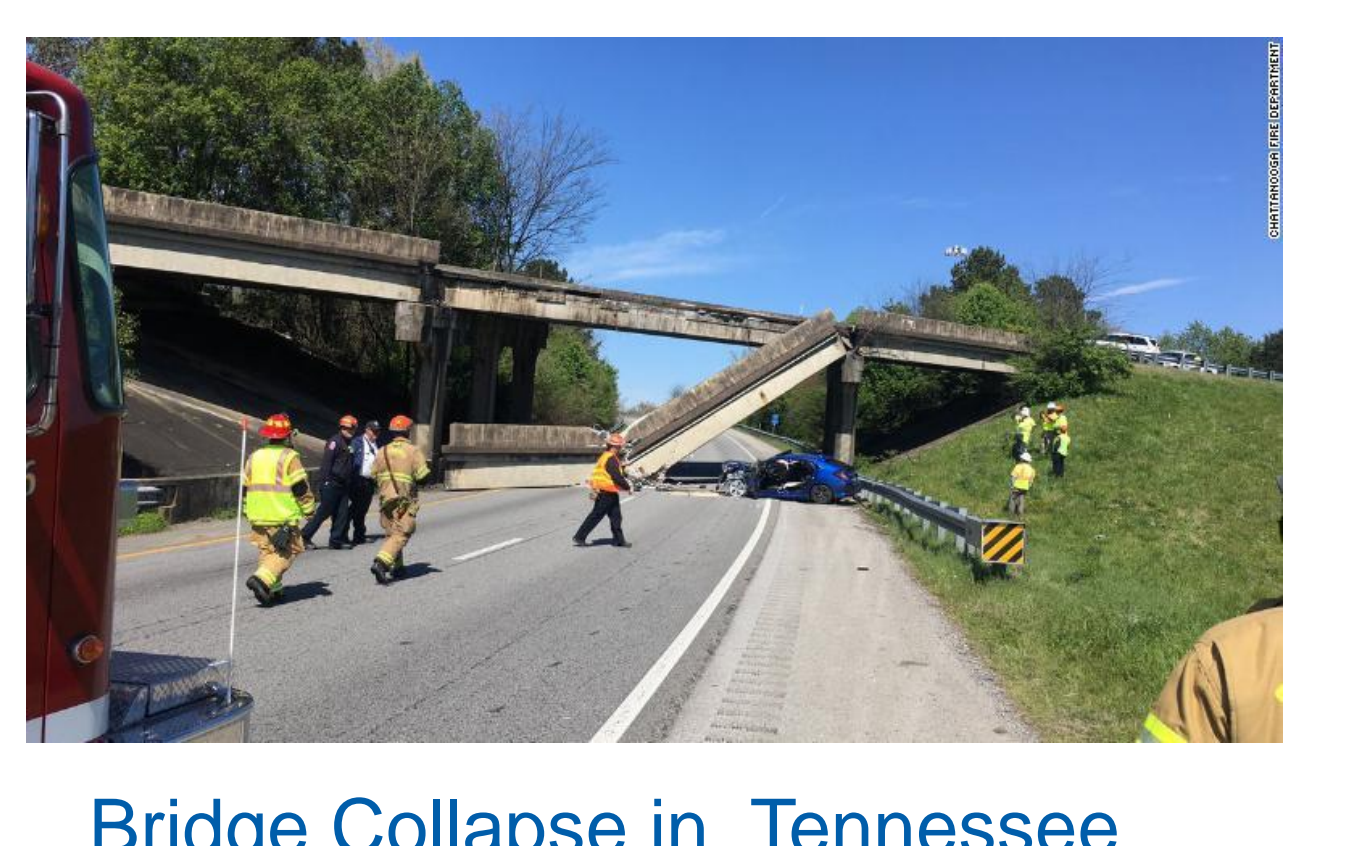

\section{RESEARCH GOAL}

To generate concrete meso-structure based on cement-aggregate proportion.

To develop a computational model as per ASR kinetics that can visualize damage propagation and predict concrete service life.

\section{ASR MODEL: GOVERNING EQUATION SETS}

Mass balance and Momentum balance: $\mathrm{D}=2.021 \times \varepsilon_{\nu o l}-0.195$

$\frac{\partial}{\partial t}\left(c_{i}\right)+\nabla \cdot\left(-D_{i} \nabla c_{i}\right)+\boldsymbol{u} \cdot \nabla c_{i}=R_{i}$

Darcy's Law:

$\frac{\partial}{\partial t}\left(\varepsilon_{p} \rho\right)+\nabla \cdot(\rho \boldsymbol{u})=Q_{m}$

$\boldsymbol{u}=-\frac{\kappa}{\mu} \nabla p$

- For Unsaturated Condition

$u=[[\text { Siloxane }],[\text { ROH }],[\text { ASR gel }],[\text { Silanol }],[H 2 O],[\text { Expanded ASR gel }]]^{T}$

$f_{[\text {Siloxane }]}=-k_{1}[$ Siloxane $][\mathrm{ROH}]$

$f_{[\mathrm{ROH}]}=-k_{1}[$ Siloxane $][\mathrm{ROH}]-k_{2}[$ Silanol $][R O H]$

$f_{[A S R \text { gel }]}=k_{1}[$ Siloxane $][R O H]+k_{2}[$ Silanol $][R O H]$
$f_{[\text {Silanol }]}=k_{1}[$ Siloxane $][\mathrm{ROH}]-k_{2}[$ Silanol $][\mathrm{ROH}]$

$f_{\left[\mathrm{H}_{2} \mathrm{O}\right]}=k_{2}[$ Silanol $][\mathrm{ROH}]-k_{3}[\mathrm{ASR}$ gel $]\left[\mathrm{H}_{2} \mathrm{O}\right]$

$f_{[\text {Expanded ASR gel }]}=k_{3}[$ ASR gel $]\left[\mathrm{H}_{2} \mathrm{O}\right]$

$$
\begin{array}{l|l}
\mathrm{D}=2.021 \times \varepsilon_{\text {vol }}-0.195 & \frac{\partial}{\partial t}\left(\varepsilon_{p} \rho\right)+\nabla \cdot(\rho \boldsymbol{u})=Q_{m} \\
\varepsilon_{\text {vol }}=\varepsilon_{\text {gel }}+\varepsilon_{\text {stress }} & \boldsymbol{u}=-\frac{\kappa}{\mu} \nabla p \\
\varepsilon_{\text {gel }}=\frac{\rho_{\text {gel }}}{\rho_{c}+\rho_{\text {gel }}} & Q_{m}=0.018 \times f_{[H 2 O]} \\
\rho_{\text {gel }}=[\text { Expanded ASR gel }] \times M_{\text {gel }} & \varepsilon_{\text {stress }}=\varepsilon_{11}+\varepsilon_{22}+\varepsilon_{33} \\
\frac{\partial}{\partial t}(u)+\nabla \cdot\left(-D_{\text {gel }} \nabla[\text { ASR gel }]\right)+\boldsymbol{u} \cdot \nabla u=f(u) & \\
\hline
\end{array}
$$

$\square \mathrm{i}=$ Denotes Each Species, respectively प $\mathrm{C}=$ Concentration of species

$\square \mathrm{Di}=$ Diffusion Coefficient

$\square \mathrm{u}=$ Velocity Field

$\square, f(u)=$ Source Term

$\square \mathrm{Qm}=$ Discharge

$\checkmark \mathrm{P}=$ Developed Pore Pressure

$\square=$ Damage Function

\section{PREDICTIVE MODEL}

A 200mm²00mm Concrete block was considered.
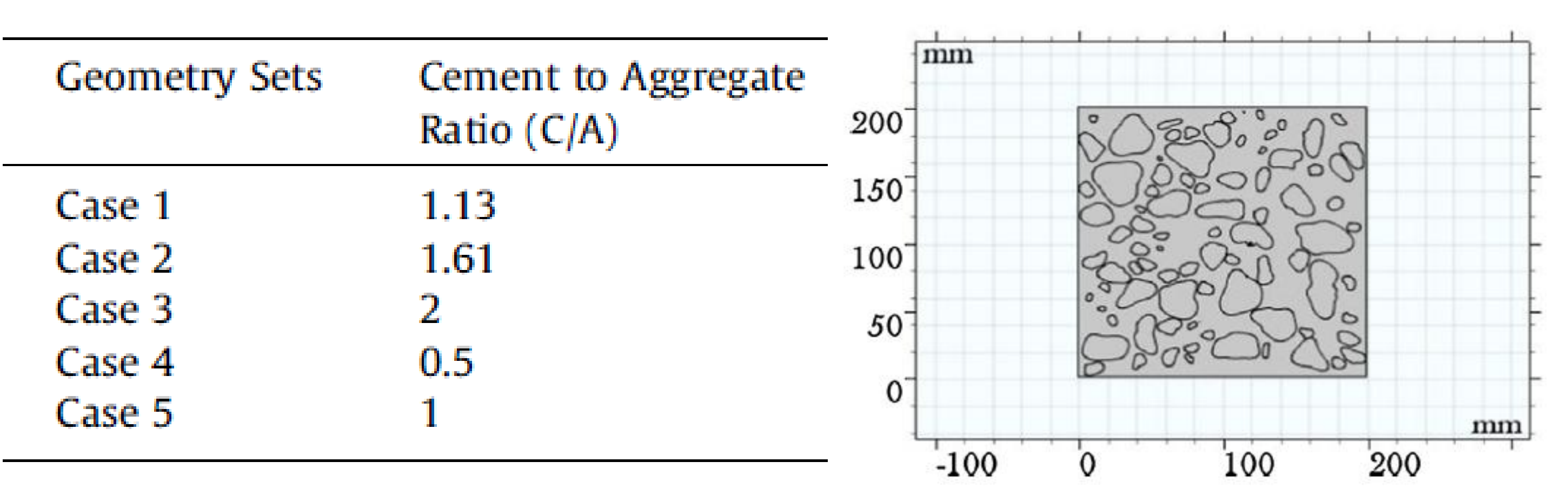

Damage Function: $D= \begin{cases}0 & \text { when }, \varepsilon_{\text {gel }} \leq 0.1 \% \\ w & \text { when }, \varepsilon_{\text {gel }}>0.1 \%\end{cases}$

For validation predictive model was run for 400 days
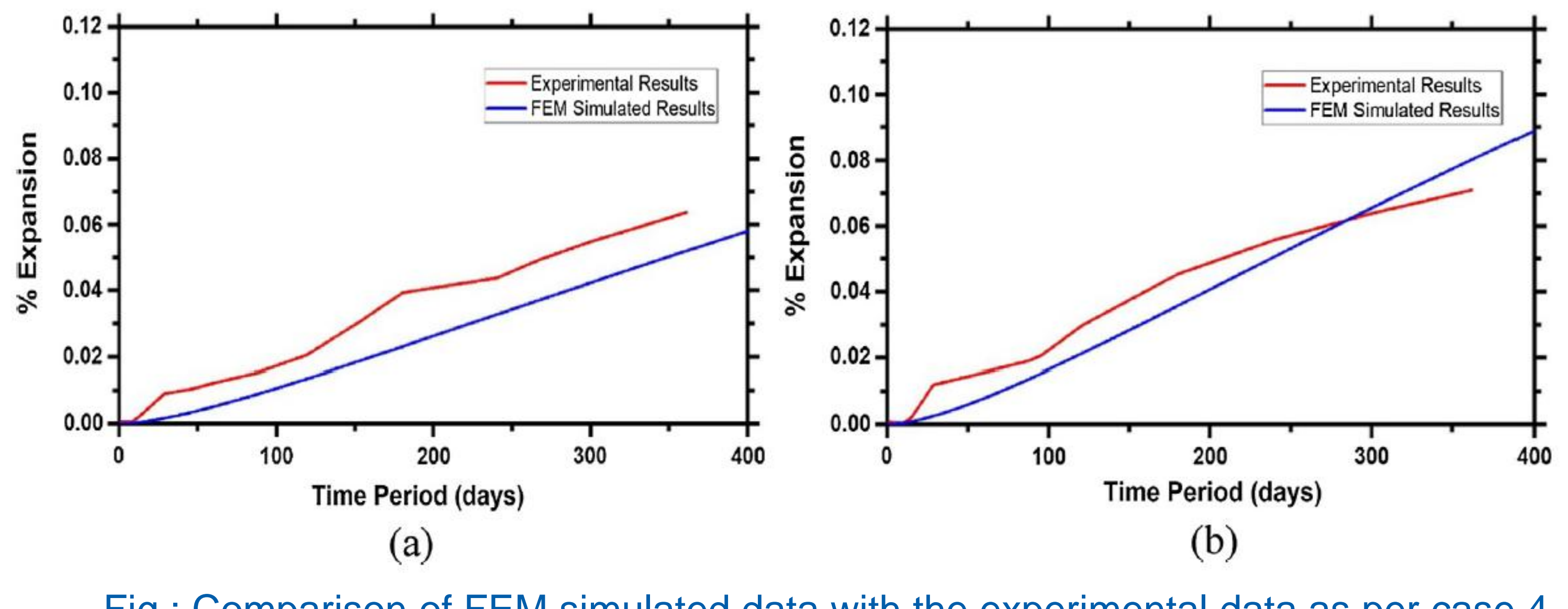

Fig: : Comparison of FEM simulated data with the experimental data as per case 4
(C/A: 0.5$):$ (a) 20 degC ROH $0.8 \mathrm{~mol} / \mathrm{m} 3$, (b) 20 degC ROH $1.2 \mathrm{~mol} / \mathrm{m} 3$

\section{RESULTS \& DISCUSSIONS*}

A 30 years simulation period as well as 10 case studies corresponding to concrete domain and $\mathrm{ROH}$ concentration, provide an in depth view of concrete degradation with the progression of time.
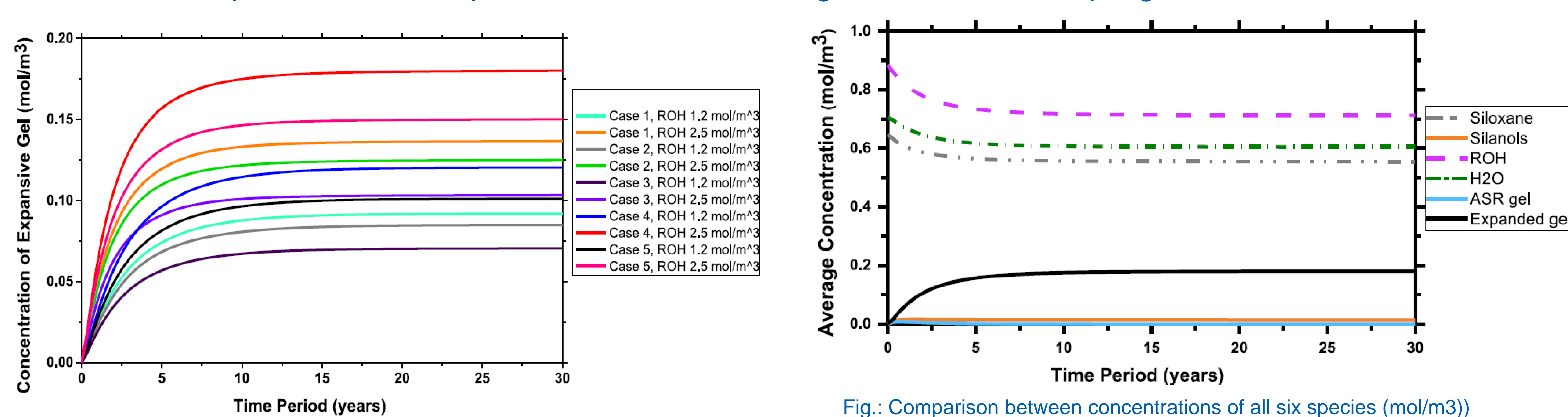

Fig.: Concentration of expansive ASR gel (mol/m3)

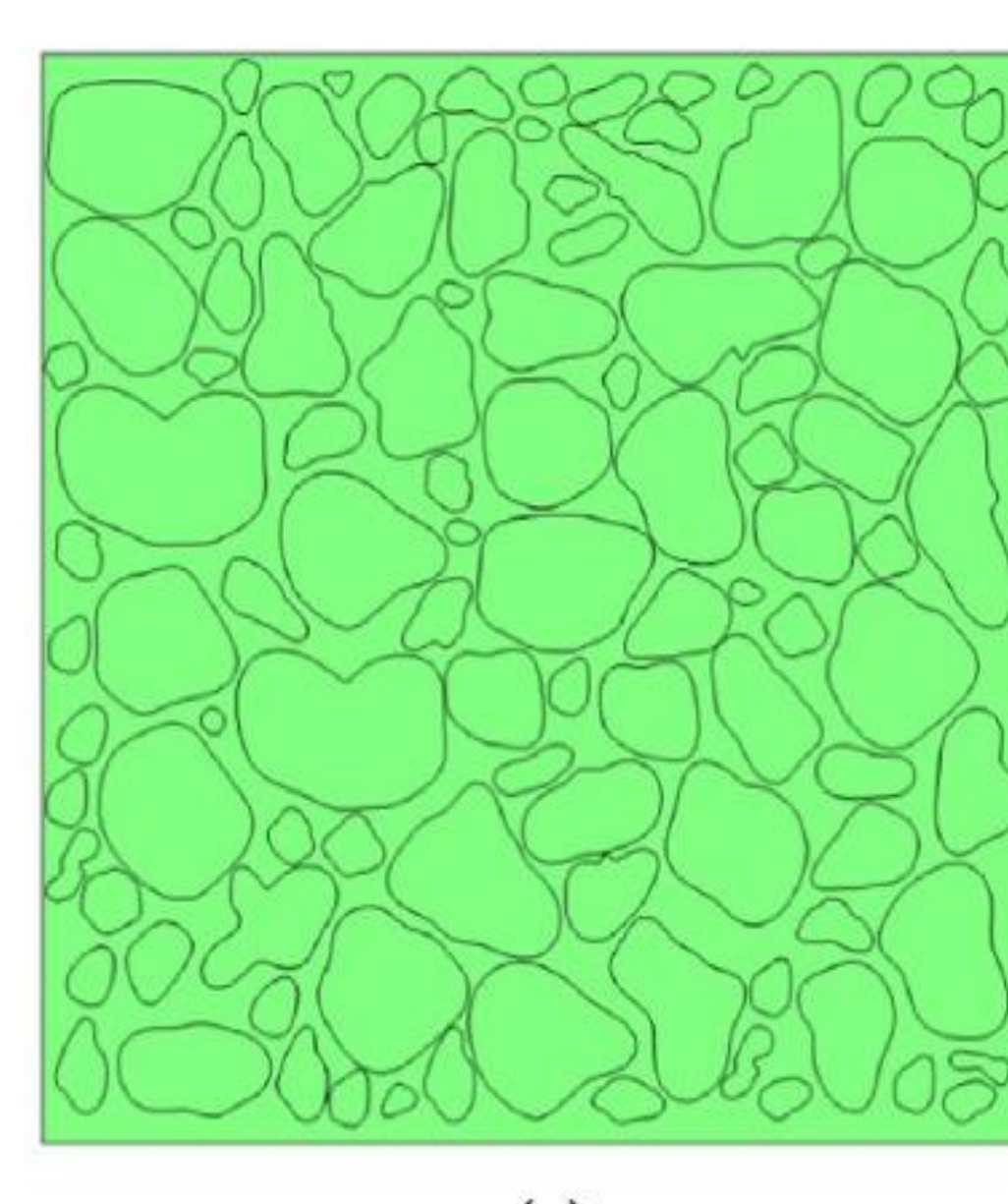

(a)
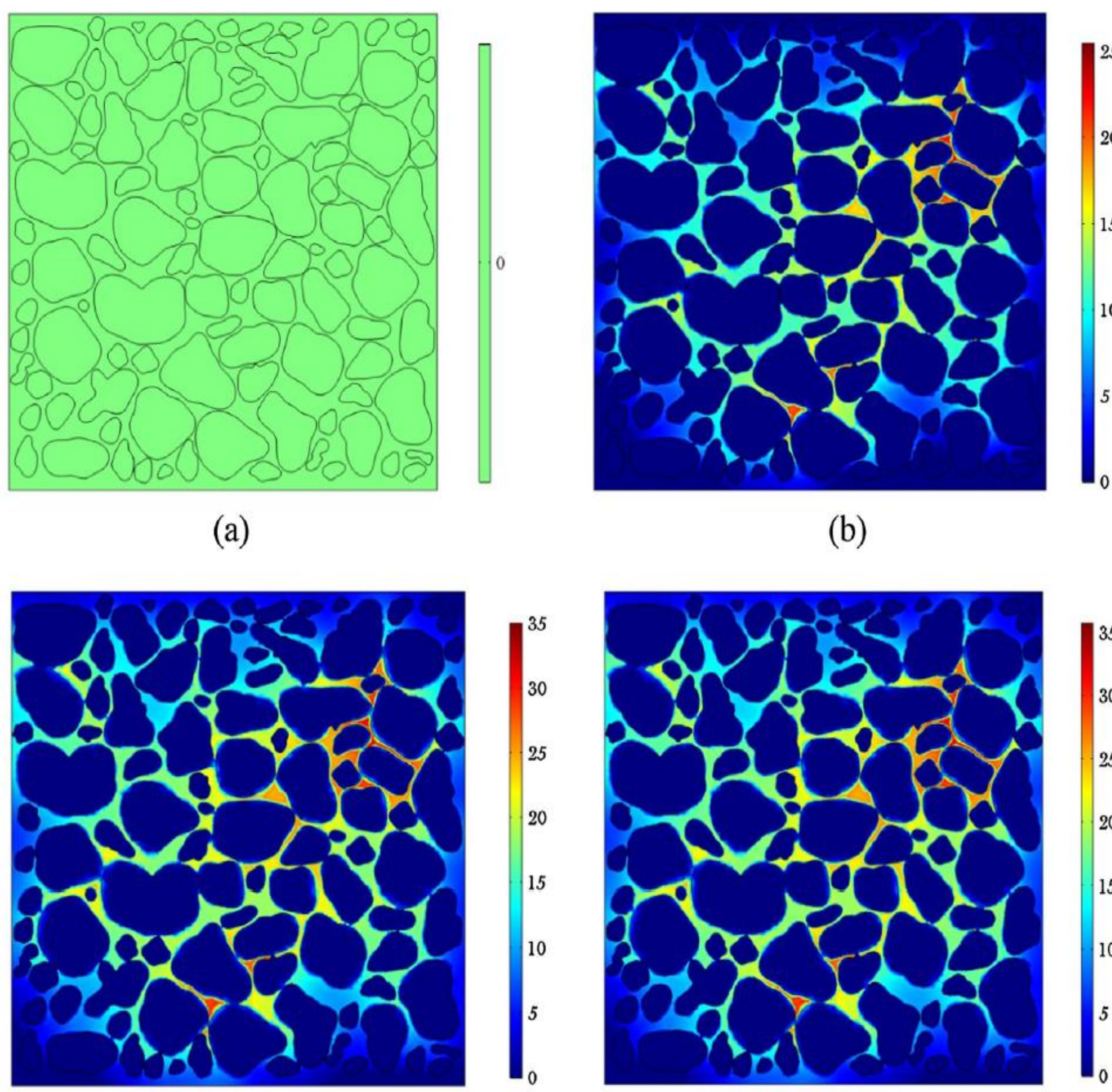

(b)

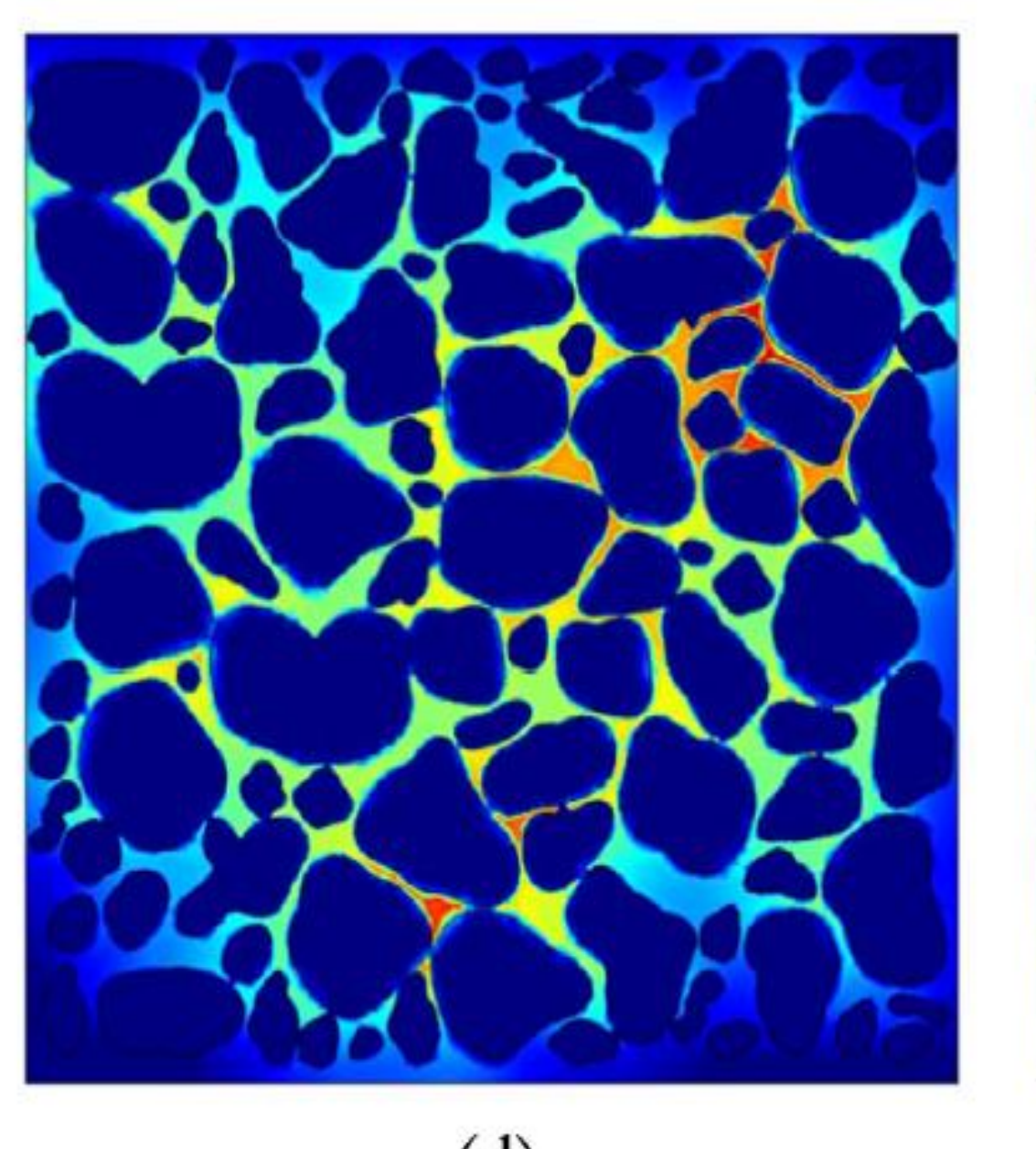

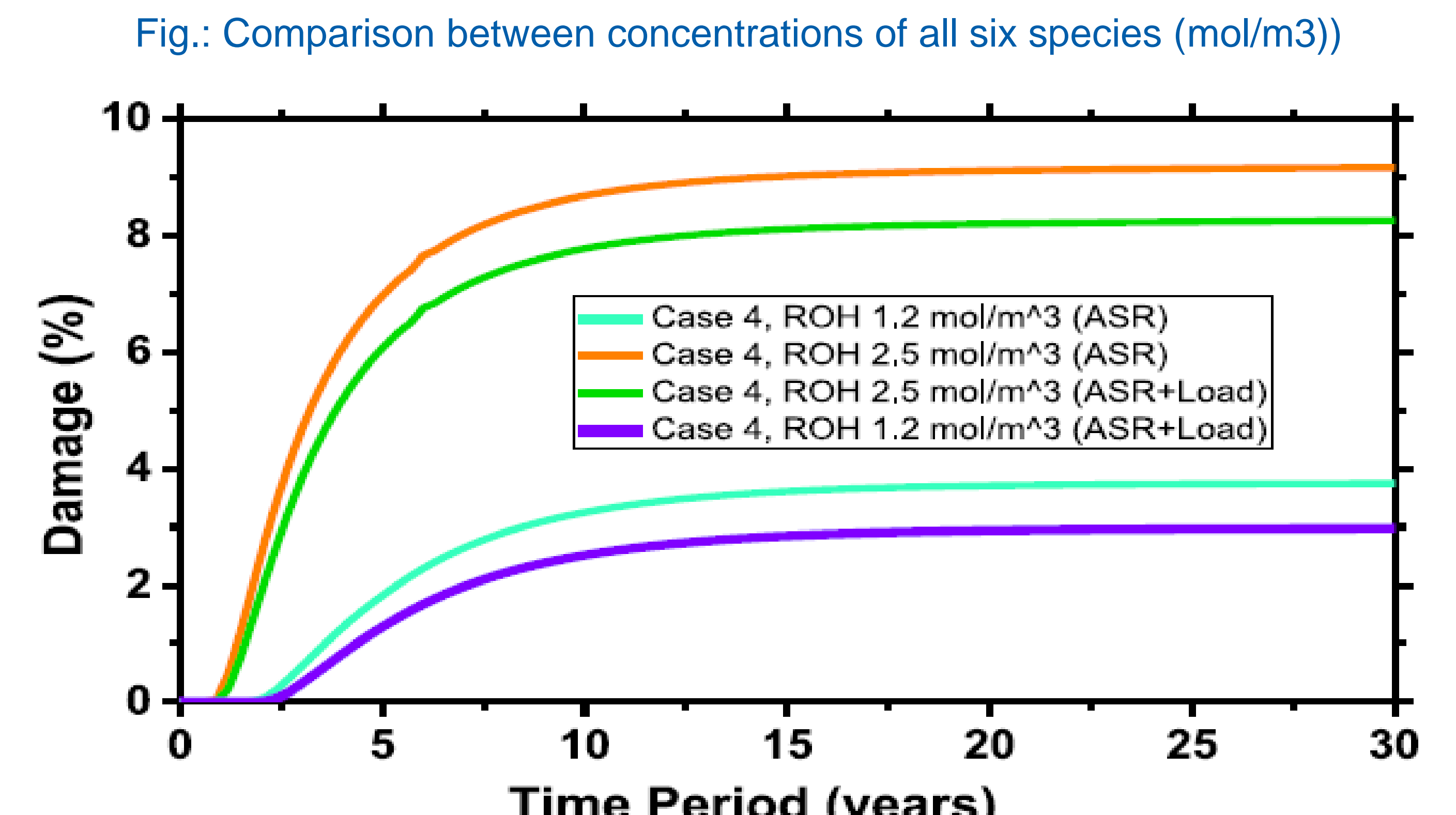

Fig. Percentage of damage in concrete domain for case 4 .
Fig.: Damage in concrete domain due to ASR gel case 4 with ROH of 1.2

HEIGHLIGHTS

An effective tool to predict service life of concrete.

Applicable to any concrete structures, i.e., Pavement. Bridges, Dams. Nuclear reactor etc.

Can act as a guideline for government or construction industries.

Potentially minimize time consuming and costly lab tests.

FUTURE GOALS

$\checkmark$ Create Database from developed model based on case study

Develop an artificial neural network (ANN) interface to predict service life.. *Rahman, Md. Asif, Yang Lu, "A Time-Dependent Chemo-Mechanical Analysis Of Alkali-Silica Reaction For The Disparate Geometry Of Concrete Meso-
Structure," Journal of Construction and Building Materials, Volume 211, 30 June 2019, Pages 847-857. 